\title{
Implantable device-related endocarditis detected by point-of-care echocardiography in a patient with dyspnea and chest pain
}

\author{
Alfonso Sforza, ${ }^{1}$ Maria Viviana Carlino, ${ }^{1}$ Mario Guarino, ${ }^{1}$ Giuliano De Stefano, ${ }^{2}$ Maria Immacolata Arnone, ${ }^{2}$ \\ Stefania Auciello, ${ }^{2}$ Andrea D’Amato, ${ }^{2}$ Costantino Mancusi ${ }^{2}$ \\ ${ }^{1}$ Emergency Department, CTO Hospital, Naples; ${ }^{2}$ Hypertension Research Center, UOC Emergency Medicine, Federico II \\ University Hospital, Naples, Italy
}

\begin{abstract}
We describe a case of a 70-year-old man who presented to the emergency department with dyspnea and chest pain. He had history of atrial fibrillation, chronic obstructive pulmonary disease, prosthetic mitral valve and pacemaker implantation. He had a sepsis related to multiple bilateral septic emboli pointed out by chest computed tomography scan. Point of care echocardiography showed a pacemaker-related endocarditis with large vegetation on the right ventricular lead. The patient started empiric antibiotic therapy and he was addressed to complete hardware removal.
\end{abstract}

\section{Introduction}

Septic pulmonary embolism (SPE) is an uncommon disorder that generally presents with an insidious onset of fever, respiratory symptoms and lung infiltrates. Clinical and radiologic features at presentation are usually nonspecific and the diagnosis of this disorder is frequently delayed. ${ }^{1}$

Correspondence: Costantino Mancusi, Hypertension Research Center, Federico II University Hospital, via Pansini 5 80131, Naples, Italy.

Tel.: +39.081.7462013. E-mail: costantino.mancusi@unina.it

Key words: Point-of-care ultrasound; emergency department; septic pulmonary embolism; pacemaker endocarditis.

Contributions: AS, MVC conceived the paper and wrote the manuscript; CM, MG, MIA, SA, GDS, AD'A contributed to the discussion and edited the manuscript.

Conflicts of interest: the authors declare no potential conflict of interest.

Received for publication: 5 June 2018.

Revision received: 4 September 2018.

Accepted for publication: 19 September 2018.

This work is licensed under a Creative Commons Attribution NonCommercial 4.0 License (CC BY-NC 4.0).

(C) Copyright A. Sforza et al., 2018

Licensee PAGEPress, Italy

Italian Journal of Medicine 2018; 12:273-275

doi:10.4081/itjm.2018.1050

\section{Case Report}

A 70-year-old man with history of atrial fibrillation episodes, chronic obstructive pulmonary disease, prosthetic biological mitral valve and pacemaker implantation some years ago, presented to the Emergency Department with chest pain, fever and dyspnea. He was on treatment with amiodarone, ramipril, furosemide, bisoprolol, inhaled medications and edoxaban. At admission blood pressure was $130 / 70 \mathrm{mmHg}$, heart rate was 80 beats/min, oxygen saturation was $85 \%\left(\mathrm{FiO}_{2} 21 \%\right)$ with tachypnea (26 breaths/minute) and body temperature was $37.7^{\circ} \mathrm{C}$. Chest examination revealed diffuse decreased vesicular murmur with crackles in the bases. Cardiovascular examination revealed augmented first heart sound, normal peripheral pulses and no edema. Arterial blood gas analysis revealed moderate hypoxemia. Electrocardiogram showed non-specific abnormalities. Chest X-ray showed non-specific opacities in the lower lungs (Figure 1). Results of blood test were consistent with a sepsis (Table 1). The patient underwent chest computed tomography (CT) scan that revealed multiple nodules consistent with the diagnosis of septic emboli (Figure 2). Point of care echocardiography was done following a standard protocol.,.$^{2,3}$ Lung ultrasound revealed a diffuse B-profile pattern. Focus cardiac ultrasound showed a pacemaker lead vegetation with a maximum diameter of $1.9 \mathrm{~cm}$ (Figure 3 ) confirmed by transesophageal echocardiography. The patient started empiric antibiotic therapy with ampicillin and gentamicin after two sets of blood cultures had been obtained and he was referred to the division of infectious diseases with availability of cardiac surgery for pacemaker lead removal. One blood 
culture in the ED was positive for Escherichia coli infection.

\section{Discussion}

Historically, SPE has been associated with risk factors such as intravenous (IV) drug use, pelvic thrombophlebitis and suppurative processes in the head and neck. However, increasing use of indwelling catheters and devices as well as increasing numbers of immunocompromised patients have changed the epidemiology and clinical manifestations of this disease. ${ }^{1}$ Historically, SPE was most commonly associated with Lemierre's syndrome, post-partum septic pelvic thrombophlebitis and right-sided infective endocarditis (IE) in IV drug users. Within the last decade, attention has been drawn to changes in the epidemiology, including the importance of infected vascular catheters and implantable devices and more recently septic thrombophlebitis due to contiguous deep soft tissue or bone infection of the extremities. ${ }^{4}$ As showed in a recent study, sources of infection in-

Table 1. Laboratory tests.

\begin{tabular}{|c|c|c|}
\hline Parameter & Value & Unit of measure \\
\hline Red blood cells & 3.62 & $\mathrm{x} 10^{6} / \mathrm{mL}$ \\
\hline Hemoglobin & 10.1 & $\mathrm{~g} / \mathrm{dL}$ \\
\hline Hematocrit & 31.2 & $\%$ \\
\hline Platelets & 240 & $\mathrm{x} 10^{3} / \mathrm{mL}$ \\
\hline White blood cells & 46.49 & $\mathrm{x} 10^{3} / \mathrm{mL}$ \\
\hline Neutrophils & 44.89 & $\mathrm{x} 10^{3} / \mathrm{mL}$ \\
\hline Glucose & 134 & $\mathrm{mg} / \mathrm{dL}$ \\
\hline Urea & 63 & $\mathrm{mg} / \mathrm{dL}$ \\
\hline Creatinine & 2.04 & $\mathrm{mg} / \mathrm{dL}$ \\
\hline Sodium & 145 & $\mathrm{mEq} / \mathrm{L}$ \\
\hline Potassium & 3.2 & $\mathrm{mEq} / \mathrm{L}$ \\
\hline Calcium & 8.1 & $\mathrm{mg} / \mathrm{dL}$ \\
\hline Albumin & 2.6 & $\mathrm{~g} / \mathrm{dL}$ \\
\hline Aspartate aminotransferase & 171 & $\mathrm{UI} / \mathrm{L}$ \\
\hline Alanine aminotransferase & 119 & $\mathrm{UI} / \mathrm{L}$ \\
\hline Bilirubin total & 2.96 & $\mathrm{mg} / \mathrm{dL}$ \\
\hline Bilirubin direct & 2.77 & $\mathrm{mg} / \mathrm{dL}$ \\
\hline International normalized ratio & 2.62 & - \\
\hline Activated partial thromboplastin time & 37.1 & $\sec$ \\
\hline C-reactive protein & 213.2 & $\mathrm{mg} / \mathrm{L}$ \\
\hline Procalcitonin & 52.5 & $\mathrm{ng} / \mathrm{mL}$ \\
\hline Pro-BNP & 270.7 & $\mathrm{pg} / \mathrm{mL}$ \\
\hline
\end{tabular}

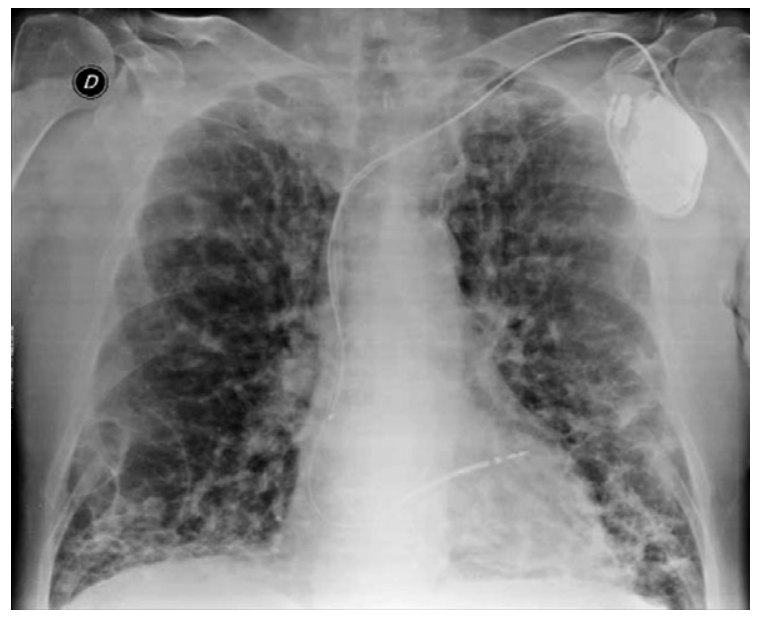

Figure 1. Chest X-ray showing non-specific opacities in the lower lungs.

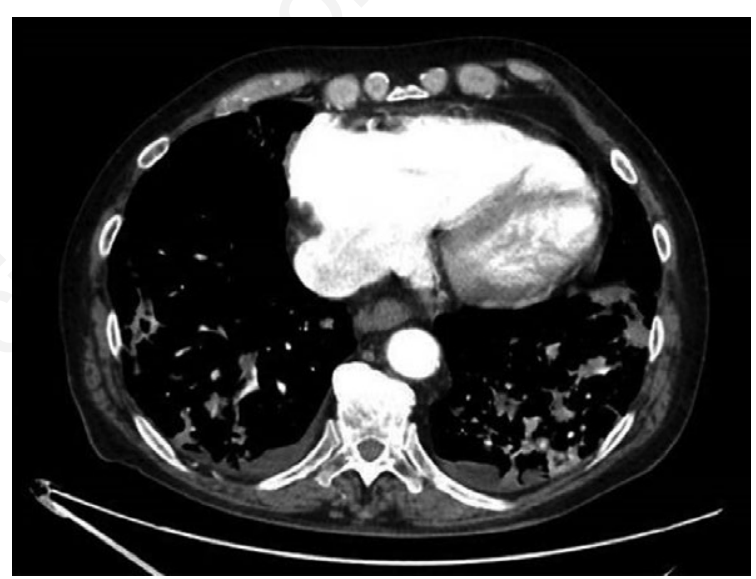

Figure 2. Chest computed tomography-scan showing multiple septic emboli in both lungs.

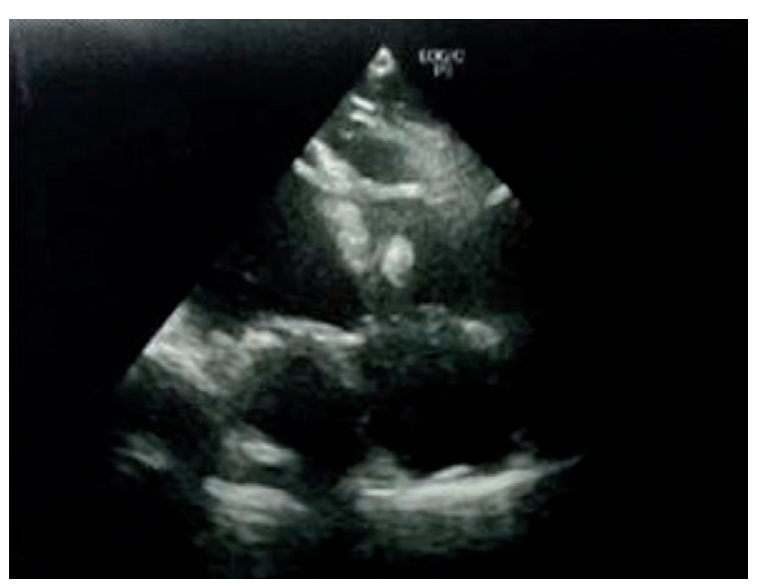

Figure 3. Point-of-care echocardiography, apical 4chamber view showing a pacemaker-related endocarditis with large vegetation on the right ventricular lead. 
cluded skin and soft tissue (44\%), IE (27\%) and infected peripheral deep venous thrombosis $(17 \%)$, in particular, in $85 \%$ of cases there was Staphylococcus aureus bacteremia. ${ }^{4}$ Infection of cardiovascular implantable electronic devices, in particular lead endocarditis, is an emerging problem with significant morbidity, mortality and health care costs. Staphylococcal species predominate as the causative organisms. Diagnosis is reliably made by blood cultures and transesophageal echocardiography. ${ }^{5}$ Transthoracic echocardiography is recommended as the firstline imaging modality in suspected infective endocarditis. ${ }^{6}$ Point-of-care ultrasound (POCUS) has been reported to diagnose both right- and left-sided $\mathrm{IE}^{7,8}$ and, for this reason, it is convincible that it can be useful also to detect infection of cardiovascular implantable electronic devices. It must be clear that a normal echocardiographic examination (included negative transesophageal echocardiography) does not rule out cardiac device-related IE. High clinical suspicion is fundamental especially in this setting of patients. The results of the first blood culture done in ED should be interpreted with caution due to the fact that Escherichia coli is an unusual bacterium for IE and could be also a contaminant. The management of cardiac device-related IE consists of prolonged antibiotic therapy and complete hardware removal. Percutaneous extraction is recommended in most patients with device infection, even those with vegetation $>10$ $\mathrm{mm}$. Surgical extraction may be considered in patients with large vegetations $(>20 \mathrm{~mm})$ and should be considered if percutaneous extraction is incomplete or impossible or when there is associated severe destructive tricuspid IE. ${ }^{6}$

\section{Conclusions}

SPE is a possible complication of right-sided IE. ${ }^{1}$ POCUS has an important role in speeding up the diagnosis that must be suspected, particularly in the presence of implantable devices. $^{7}$

\section{References}

1. Cook RJ, Ashton RW, Aughenbaugh GL, Ryu JH. Septic pulmonary embolism: presenting features and clinical course of 14 patients. Chest 2005;128:162-6.

2. Sforza A, Mancusi C, Carlino MV, et al. Diagnostic performance of multi-organ ultrasound with pocket-sized device in the management of acute dyspnea. Cardiovasc Ultrasound 2017;15:16.

3. Carlino MV, Paladino F, Sforza A, et al. Assessment of left atrial size in addition to focused cardiopulmonary ultrasound improves diagnostic accuracy of acute heart failure in the Emergency Department. Echocardiography 2018;35:785-91.

4. Goswami U, Brenes JA, Punjabi GV, et al. Associations and outcomes of septic pulmonary embolism. Open Respir Med J 2014;8:28-33.

5. Athan E. The characteristics and outcome of infective endocarditis involving implantable cardiac devices. Curr Infect Dis Rep 2014;16:446.

6. Habib G, Lancellotti P, Antunes MJ, et al. 2015 ESC Guidelines for the management of infective endocarditis. Eur Heart J 2015;36:3075-128.

7. Seif D, Meeks A, Mailhot T, Perera P. Emergency department diagnosis of infective endocarditis using bedside emergency ultrasound. Crit Ultrasound J 2013;5:1.

8. Bugg CW, Berona K. Point-of-care ultrasound diagnosis of left-sided endocarditis. West J Emerg Med 2016; 17:383. 